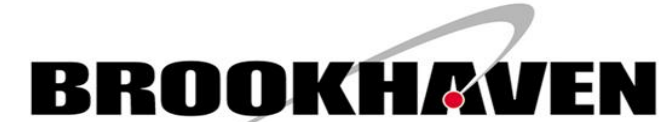 \\ NATIONAL LABORATORY
}

BNL-108080-2015-CP

\section{Doping-controlled Coherent Electron-Phonon Coupling in Vanadium Dioxide}

\author{
Kannatassen Appavoo \\ Kannatassen Appavoo ${ }^{1,2}$, Bin Wang ${ }^{3}$, Joyeeta $\mathrm{Nag}^{3}$, Matthew Y. Sfeir ${ }^{2}$, \\ Sokrates T. Pantelides ${ }^{3},{ }^{4}$, Richard F. Haglund ${ }^{13}$ \\ ${ }^{1}$ Interdisciplinary Materials Science, Vanderbilt University, Nashville, TN \\ ${ }^{2}$ Center for Functional Nanomaterials, Brookhaven National Laboratory, Upton, NY \\ ${ }^{3}$ Department of Physics and Astronomy, Vanderbilt University, Nashville, TN \\ ${ }^{4}$ Materials Science and Technology Division, Oak Ridge National Laboratory, Oak Ridge, TN \\ ${ }^{5}$ Department of Electrical Engineering and Computer Science, Vanderbilt University, Nashville, TN
}

\author{
Center for Functional Nanomaterials \\ Brookhaven National Laboratory
}

\section{U.S. Department of Energy Office of Basic Energy Sciences}

Notice: This manuscript has been authored by employees of Brookhaven Science Associates, LLC under Contract No. DE-AC0298CH10886 with the U.S. Department of Energy. The publisher by accepting the manuscript for publication acknowledges that the United States Government retains a non-exclusive, paid-up, irrevocable, world-wide license to publish or reproduce the published form of this manuscript, or allow others to do so, for United States Government purposes.

This preprint is intended for publication in a journal or proceedings. Since changes may be made before publication, it may not be cited or reproduced without the author's permission. 


\section{DISCLAIMER}

This report was prepared as an account of work sponsored by an agency of the United States Government. Neither the United States Government nor any agency thereof, nor any of their employees, nor any of their contractors, subcontractors, or their employees, makes any warranty, express or implied, or assumes any legal liability or responsibility for the accuracy, completeness, or any third party's use or the results of such use of any information, apparatus, product, or process disclosed, or represents that its use would not infringe privately owned rights. Reference herein to any specific commercial product, process, or service by trade name, trademark, manufacturer, or otherwise, does not necessarily constitute or imply its endorsement, recommendation, or favoring by the United States Government or any agency thereof or its contractors or subcontractors. The views and opinions of authors expressed herein do not necessarily state or reflect those of the United States Government or any agency thereof. 


\title{
Doping-controlled Coherent Electron-Phonon Coupling in Vanadium Dioxide
}

\author{
Kannatassen Appavoo ${ }^{1,2 *}$, Bin Wang ${ }^{3}$, Joyeeta $\mathrm{Nag}^{3}$, Matthew Y. Sfeir ${ }^{2}$, \\ Sokrates T. Pantelides ${ }^{3,4,5}$, Richard F. Haglund ${ }^{1,3}$ \\ ${ }^{I}$ Interdisciplinary Materials Science, Vanderbilt University, Nashville, TN 37234 \\ ${ }^{2}$ Center for Functional Nanomaterials, Brookhaven National Laboratory, Upton, NY 11973 \\ ${ }^{3}$ Department of Physics and Astronomy, Vanderbilt University, Nashville, TN 37235 \\ ${ }^{4}$ Materials Science and Technology Division, Oak Ridge National Laboratory, Oak Ridge, TN 37831 \\ ${ }^{5}$ Department of Electrical Engineering and Computer Science, Vanderbilt University, Nashville, TN 37235 \\ *kappavoo@bnl.gov
}

\begin{abstract}
Broadband femtosecond transient spectroscopy and density functional calculations reveal that substitutional tungsten doping of a $\mathrm{VO}_{2}$ film changes the coherent phonon response compared to the undoped film due to altered electronic and structural dynamics.

OCIS codes: (320.7130) Ultrafast processes in condensed matter; (300.6250) Spectroscopy, condensed matter
\end{abstract}

To design and fabricate optoelectronic and photonic devices operating at terahertz speeds, it is crucial to understand how ultrafast optical pulses couple to the microscopic degrees of matter. Recently, coherent phonons quantized atomic lattice vibrations - have attracted considerable interest as a tool to probe [1] and modify [2] nonequilibrium properties of matter. Here we demonstrate how elemental doping - even at 2 at. $\%$ - can provide a viable route to modifying coherent phonon modes, which causes drastic changes in the energy requirements in the ultrafast switching properties of a phase-change material (PCM), vanadium dioxide.

Vanadium dioxide exhibits a reversible solid-solid phase transformation that combines an insulator-to-metal transition (IMT) with a structural phase transition from the monoclinic insulating (M1) phase to a rutile, tetragonal (R) form at near-room temperature $\left(68^{\circ} \mathrm{C}\right)$. When triggered optically, its transformation, clocked by its band gap collapse, occurs within 100 fs [3]. When the phase-transformation is driven well above the fluence threshold for the IMT or by charge injection, a catastrophic phonon collapse also occurs [1, 4].

Here, we demonstrate how elemental doping modulates the delicate electron-phonon-orbital coupling that exists in this class of phase-change materials. As a proof-of-principle, we compare pristine $\mathrm{VO}_{2}$ thin film to its tungstendoped counterpart, both grown by pulsed laser deposition to about $60 \mathrm{~nm}$ thick [5]. Fig. 1 shows a representative broadband ultrafast $\mathrm{VO}_{2}$ response when excited at near-threshold. Within the first picosecond, one observes strong

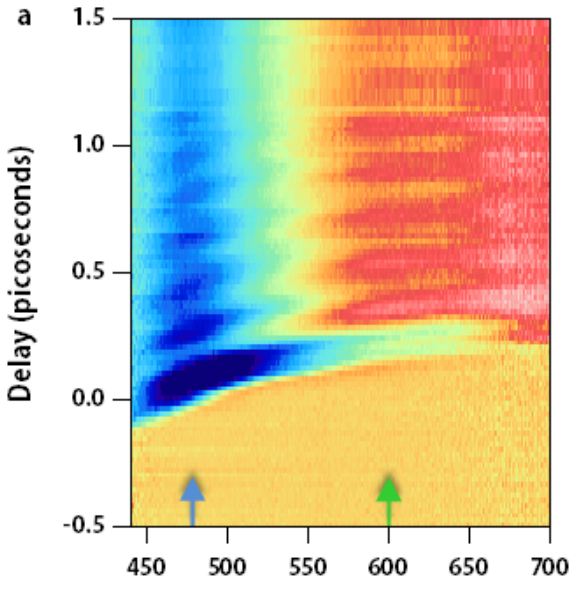

Wavelength $(\mathrm{nm})$

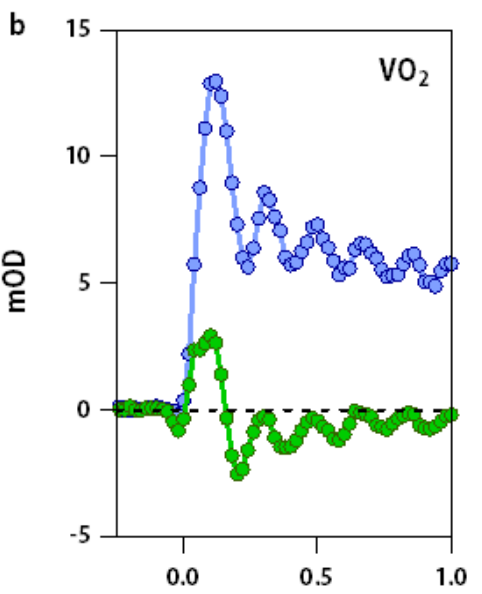

Delay (ps)

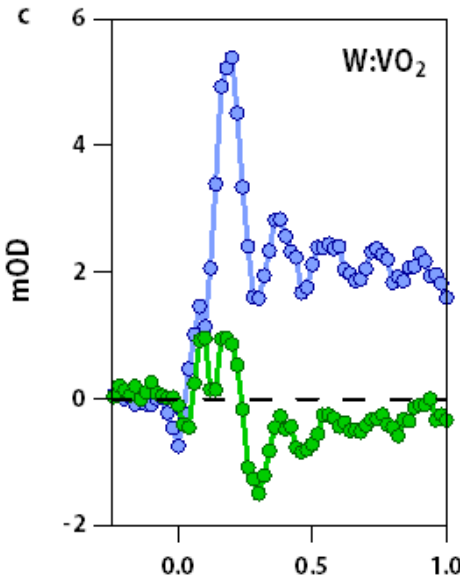

Delay (ps)

Figure 1. (a) Representative ultrafast broadband absorption response of $\mathrm{VO}_{2}$ at near threshold. Note the data is not chirp corrected. (b, c) Kinetic traces at $\sim 425 \mathrm{~nm}$ and $\sim 600 \mathrm{~nm}$ for pristine $\mathrm{VO}_{2}$ and $\mathrm{W}$-doped $\mathrm{VO}_{2}$ plotted on the same $1 \mathrm{ps}$ timescale and optically excited at the same energy fluence. Note the change in oscillatory behaviour, with a dominant $6 \mathrm{THz}$ mode for pristine $\mathrm{VO}_{2}$ while an additional $4 \mathrm{THz}$ mode is present for the $\mathrm{W}: \mathrm{VO}_{2}$, associated with the $\mathrm{W}$-ion vibration. 
modulation of the coherent phonon modes that decays rapidly. Kinetic traces at key wavelengths corresponding to the O2p-V3d transition bands, accompanied by Fourier transform reveals the presence of the strong $6 \mathrm{THz}$ characteristic mode in both films. Additionally, the $\mathrm{W}: \mathrm{VO}_{2}$ shows an additional frequency component at $4 \mathrm{THz}$. As revealed by density functional theory, this mode corresponds to the $\mathrm{W}$ vibration as it couples to the surrounding $\mathrm{VO}_{2}$ unit cells, thus upsetting the charge ordering. Together with two additional electrons from the $\mathrm{W}^{6+}$ ions compared to the $\mathrm{V}^{4+}$, this modifies the energy requirements for switching $\mathrm{VO}_{2}$. Fluence-dependent measurement shows that this 2 at.\% doping level reduces the threshold by about $1 \mathrm{~mJ} / \mathrm{cm}^{2}$.

In order to understand how this doping affects the electronic properties of the $\mathrm{VO}_{2}$, we also study their broadband ultrafast response in the near-infrared region, which is close to the $\mathrm{VO}_{2}$ band gap $(0.69 \mathrm{eV})$. Figure 2 displays the $\mathrm{VO}_{2}$ response when triggered at the pristine $\mathrm{VO}_{2}$ near-switching threshold (semiconductor like response). Two important differences are observed: (i) the $\mathrm{W}: \mathrm{VO}_{2}$ domains have already switched below the fluence needed to switch pristine $\mathrm{VO}_{2}$ and (ii) the change in $\mathrm{W}: \mathrm{VO}_{2}$ absorption (transmission contrast) is less than that of the pristine $\mathrm{VO}_{2}$. The former can be attributed to the additional electrons in the conduction band in $\mathrm{W}: \mathrm{VO}_{2}$ while the decrease in optical contrast is potentially due to fast electron-hole recombination, following ultrafast excitation. Density functional calculations show that the additional charges (either holes or electrons) are localized near the Wdopant. Thus, this enhanced electron-hole interaction can produce an overall decrease in the absorption changes.
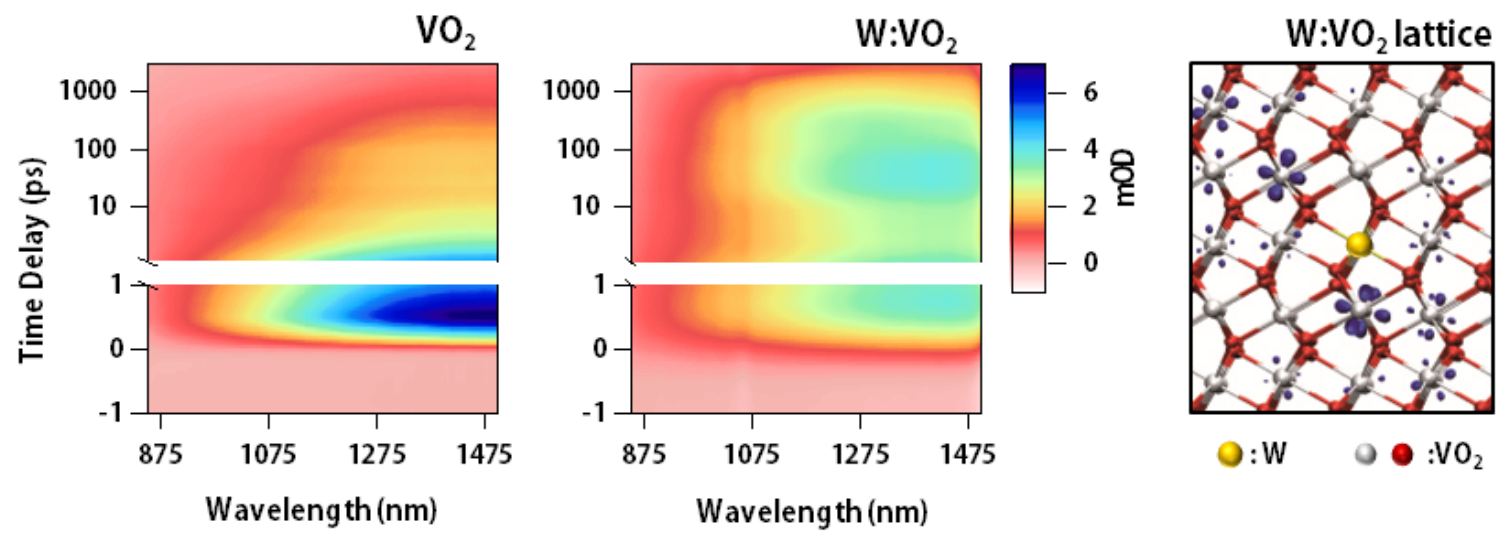

Figure 2. (a) Broadband near-IR ultrafast response of pristine and $\mathrm{W}$-doped $\mathrm{VO}_{2}$ excited at the same optical fluence and plotted on the sam intensity scale. The $\mathrm{VO}_{2}$ film shows semiconductor-like response while the $\mathrm{W}: \mathrm{VO}_{2}$ film is already in its switched state. (b) Induced electron energy difference of the $\mathrm{W}: \mathrm{VO}_{2}$ lattice displaying that the electron is localized near the $\mathrm{W}$-ion. Similar figures were obtained with holes.

In summary, we have shown the rich ultrafast dynamics of $\mathrm{PCM} \mathrm{VO}_{2}$ and how coupling with its various microscopic degrees of freedom can be tuned by doping. More importantly, we conclude that doping, even at quite low concentrations, can affect the energy requirements for the ultrafast switching of phase-change material, modifying both its electronic and coherent phonon dynamics.

\section{Acknowledgements}

Research is carried out at the Center for Functional Nanomaterials, Brookhaven National Laboratory, which is supported by the U.S.DOE (DEAC02-98CH10886). At Vanderbilt University, support is acknowledged from the National Science Foundation (ECS-0801985, NSF ARI-R2 DMR-0963361) and the Defense Threat-Reduction Agency (HDTRA1-01-1-0047). DFT calculations were partly sponsored by DOE (DE-FG0209ER46554) and the McMinn Endowment at Vanderbilt University (STP). Work at Oak Ridge National Laboratory is supported by a contract with UT-Batelle U.S DOE (DE-AC05-00OR22725).

\section{References}

[1] Wall, S. et al. "Ultrafast changes in lattice symmetry probed by coherent phonons." Nat Commun 3, 721, (2012).

[2] Beaud, P. et al. "A time-dependent order parameter for ultrafast photoinduced phase transitions." Nat Mater 13, 923-927, (2014).

[3] Wegkamp, D. et al. "Instantaneous Band Gap Collapse in Photoexcited Monoclinic $\mathrm{VO}_{2}$ due to Photocarrier Doping." Physical Review Letters 113, 216401, (2014).

[4] Appavoo, K. et al. "Ultrafast Phase Transition via Catastrophic Phonon Collapse Driven by Plasmonic Hot-Electron Injection." Nano Letters 14, 1127-1133, (2014).

[5] Nag, J. \& Haglund, R. F., Jr. "Synthesis of vanadium dioxide thin films and nanoparticles." Journal of Physics-Condensed Matter 20, (2008). 